\title{
Anorectal malformation associated with a mutation in the P63 gene in a family with split hand-foot malformation
}

\author{
Pengjun Su • Yuhang Yuan • Ying Huang • \\ Weilin Wang $\cdot$ Zhibo Zhang
}

Accepted: 22 May 2013 / Published online: 5 June 2013

(C) The Author(s) 2013. This article is published with open access at Springerlink.com

\begin{abstract}
Purpose The aims of this study were to identify the mutation gene of a Chinese family with anorectal malformation (ARM) associated with split hand-foot malformation and to determine the spatiotemporal expression of the mutated gene during hindgut and anorectum development in human embryos.

Method A Chinese family with intrafamilial clinically variable manifestation was analyzed and primers were designed for exons 3-14 of P63, DLX5, DLX6, DAC, and HOXD13 as candidate genes and direct sequence analysis of the exons was performed. Immunohistochemical study of mutated gene in the hindgut and anorectum of human embryos of 4th-10th weeks was performed.

Result Affected individuals were found to have an $\mathrm{Arg} 227 \mathrm{Gln}$ P63 gene mutation. From the 4th-10th weeks of gestation of the human embryo, the P63-positive cells were mainly located on the epithelium of the apical urorectal septum, hindgut, and cloacal membrane. After the anorectum ruptured during the 8th week, the P63 remained strongly immunoreactive on the epithelium of the anal canal and urethra, but the mucous membrane of the rectum exhibited no reaction.

Conclusions The mutation identified strongly suggests a causal relationship between the ARM phenotype and P63. The expression of P63 was persistently active during the dynamic and incessant septation of the cloaca and hindgut, suggesting that P63 may play a pivotal role in the morphogenesis of the hindgut and anorectum.
\end{abstract}

This study was conducted at the Shengjing Hospital of China Medical University, Shenyang City, China

P. Su $\cdot$ Y. Yuan $\cdot$ Y. Huang $\cdot$ W. Wang $\cdot$ Z. Zhang $(\bowtie)$

Department of Pediatric Surgery, Shengjing Hospital, China

Medical University, Shenyang City 110004, China

e-mail: zhangzb57311@126.com
Keywords Anorectal malformation - Split hand-foot malformation $\cdot P 63 \cdot$ Human embryo $\cdot$ Hindgut/anorectum

\section{Introduction}

Anorectal malformation (ARM) is a severe, congenital anomaly that can occur either in isolated cases or in association with other congenital abnormalities. Although the aetiological factors remain largely unknown, genetic abnormalities have been reported in patients with ARM. Genetic studies of various syndromes involving ARM could provide insight into anorectal development and may be helpful in elucidating the etiology of ARM $[1,2]$.

The split hand-foot malformation (SHFM or ectrodactyly, MIM 83600) is a congenital defect of the distal limbs occurring in approximately 1 in 18,000 newborns. It is characterized by varying degrees of central longitudinal fissuring and central ray deficiency frequently associated with syndactyly, along with aplasia and/or hypoplasia of the phalanges, metacarpals, and metatarsals [3]. SHFM comprises both isolated and hereditary forms, and is linked to five distinct loci (types I-V): SHFM1 on 7q21.3-q22.1, SHFM2 on Xq26, SHFM3 on 10q24, SHFM4 on $3 \mathrm{q} 27$, and SHFM5 on $2 \mathrm{q} 31$. Possible candidate genes include DLX5/DLX6, FHL1, DAC, P63, and HOXD13 [4].

Here, we describe a Chinese family with intrafamilial, clinically variable manifestations of SHFM associated with ARM and were primarily interested in discovering hereditary factors underlying the development of both the hindgut and anorectum.

\section{Materials and methods}

This study was conducted in accordance with the World Medical Association Declaration of Helsinki and approved 
by the Ethics Committee of China Medical University. Written informed consents were obtained from each subject. Parents consented on the behalf of the patients who were under 18 years old (IV-1, IV-2).

\section{Patients}

Members of this family originate from Northeast China. The patients included the grandfather, father, and the proband. They were otherwise healthy, and there were no other patients with any anomalies in this family. All affected members underwent an extensive workup including thorough clinical evaluation and routine blood tests.

\section{Molecular analysis}

EDTA blood samples were collected from all members of the family for genetic study. DNA was extracted from leukocytes using the standard salt precipitation method. Oligonucleotide primers were designed for exons 3-14 of P63, $D L X 5, D L X 6, D A C$, and HOXD13 using Primer Premier 5.0 , and direct sequence analysis of the exons (including exon-intron boundaries) was performed. PCR was performed according to standard methods.

PCR products were excised from agarose gels, purified with TIAN gel Midi columns (TIANGEN), and sequenced with a 3730 DNA Sequencer (ABI PRISM). Sequence analysis was performed using the DNASTAR and BLAST programs.

Immunohistochemical study of P63 in the developing hindgut and anorectum

The women who were selected for this study were those who were undergoing elective terminations of unplanned pregnancies without a history of hereditary disease. Sixty phenotypically normal human embryos of 4 th-10th week gestational age were obtained from chemically induced/atraumatic curettage terminations of pregnancies. The embryos were immediately washed in cold phosphatebuffered saline (PBS; pH 7.4), then fixed in $4 \%$ buffered paraformaldehyde at $4{ }^{\circ} \mathrm{C}$ for $24 \mathrm{~h}$. The samples were dehydrated, embedded in paraffin, and sectioned sagittally at a thickness of $4 \mu \mathrm{m}$. The age of the embryos was assessed according to Carnegie staging, which is the standard reference for staging.

Sample preparation was performed according to established protocols. The endogenous peroxidase activity was blocked by incubation in $3 \% \mathrm{H}_{2} \mathrm{O}_{2}$ for $10 \mathrm{~min}$. Antigen retrieval was performed by heating the slides in $10 \mathrm{mmol} / \mathrm{L}$ citrate buffer ( $\mathrm{pH} 6.0$ ) at $98^{\circ} \mathrm{C}$ for $10 \mathrm{~min}$. The sections were treated and incubated with primary goat polyclonal anti-P63 antibody (1:100; Santa Cruz Biotechnology, Inc., Santa Cruz, A, USA) and horseradish peroxidase-conjugated secondary antibody (Santa Cruz Biotechnology, Inc). Antibody incubations were performed in PBS, supplemented with $10 \%$ rabbit serum. Primary antibody was incubated with sections at $4{ }^{\circ} \mathrm{C}$ for $16 \mathrm{~h}$. Incubation of secondary antibody was performed for $20 \mathrm{~min}$ at room temperature. The immunoreactions were visualized using 3,3P-diaminobenzidine (Sigma, UK) as a chromogen. Sections were counterstained with hematoxylin. Negative controls were performed by omitting the primary or secondary antibody or by incubation with equivalent concentrations of non-immune rabbit antiserum. Two pathologists independently reviewed the immunohistochemistry-stained slides and came to a consensus on the results.

\section{Results}

Clinical features of the affected family members

In this study, we examined eight individuals from a single Chinese family (four males and four females, Fig 1.), in which three males were affected with ARM and limb deformity. The proband (IV1) was admitted to our ward because of imperforate anus. This 2-day-old male patient was born at term by vaginal delivery from unrelated parents. Prenatal ultrasonographic investigations were unremarkable. After detailed physical examination, he was diagnosed with intermediate imperforate anus, rectourethral fistula, and penoscrotal hypospadias. In addition, the boy presented with an absence of the second and third metacarpus, phalanges of the right hand, third and fourth finger syndactyly of the left hand, and third and fourth toe syndactyly of the left foot (Fig. 2a-c). The proband's father and grandfather both suffered from low imperforate anus with a perineal fistula. In addition, the father presented with absence of the third phalanges of the right hand (Fig. 2d), and the grandfather exhibited third and fourth finger syndactyly of the right hand. The proband (IV1), now 2 years old, together with all the other individuals of the family, had none of the significant abnormalities of the face, palate, skin, teeth, hair, or nails that characterize ectrodactyly-ectodermal dysplasiacleft lip/palate (EEC) syndrome.

\section{Mutation analysis}

In this study, we analyzed the $D L X 5 / D L X 6, D A C, P 63$, and $H O X D 13$ sequences of three affected and five unaffected individuals in the Chinese family. Extensive sequencing analysis was performed and identified no mutations in the $D L X 5 / D L X 6, D A C$, and HOXD13 genes (data not shown); however, a missense mutation in the DNA-binding domain was found in exon 6 of P63: Arg227Gln $(797 G \rightarrow A)$ (Fig. 3). This mutation was not found in the five unaffected individuals in the family. Besides, we also sequenced 100 

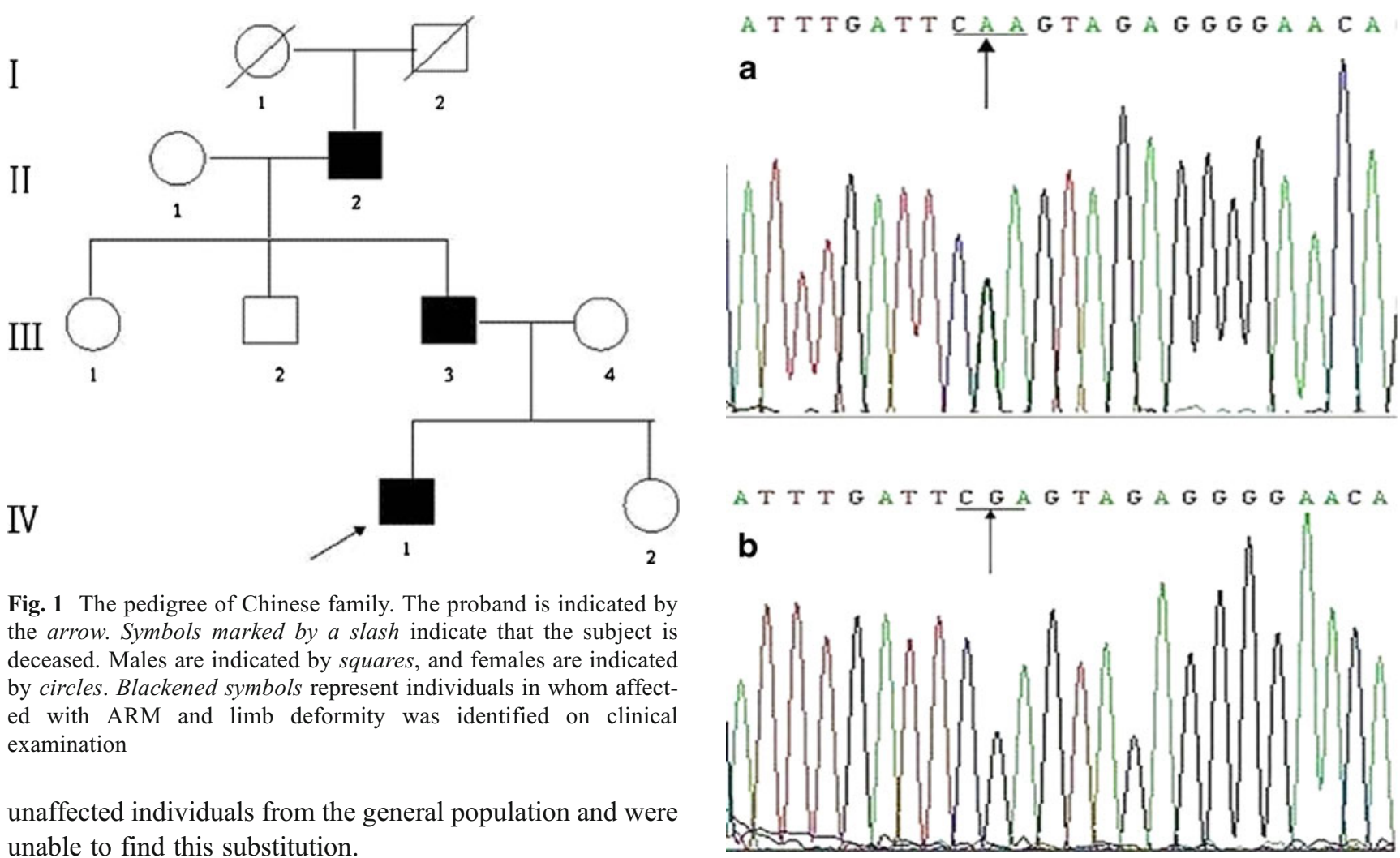

Fig. 1 The pedigree of Chinese family. The proband is indicated by the arrow. Symbols marked by a slash indicate that the subject is deceased. Males are indicated by squares, and females are indicated by circles. Blackened symbols represent individuals in whom affected with ARM and limb deformity was identified on clinical examination

unaffected individuals from the general population and were unable to find this substitution.

The expression of $P 63$ in the development of hindgut and anorectum

To further define the role of $P 63$ in hindgut/anorectal development, the ontogeny of P63 expression in the cloaca was examined via immunohistochemistry in embryos aged 4th10th week gestational age using a 4A4 pan-P63 antibody.

Fig. 3 Sequencing of exon 6 of $P 63$ for the patients with ARM and limb defects and the controls. A heterozygous missense mutation (CGA > CAA) at 797 nucleotide in exon 6 of P63 gene (R227Q) was identified in the three affected patients. Sequencing in exon 6 of P63 gene for the proband (a) and the controls (b) are indicated

In the 4th week, a typical cloaca was present. The U-shaped urorectal septum (URS) divided the cloaca into the urogenital sinus (UGS) ventrally and the primitive rectum (hindgut)

Fig. 2 The clinical features of the limbs of the proband (ac)and his father (d). a Absence of the second and third metacarpus, phalanges of the right hand; $\mathbf{b}$ third and forth finger syndactyly of the left hand; $\mathbf{c}$ third and fourth toe syndactyly of the left foot; $\mathbf{d}$ absence of the third phalanges of the right hand

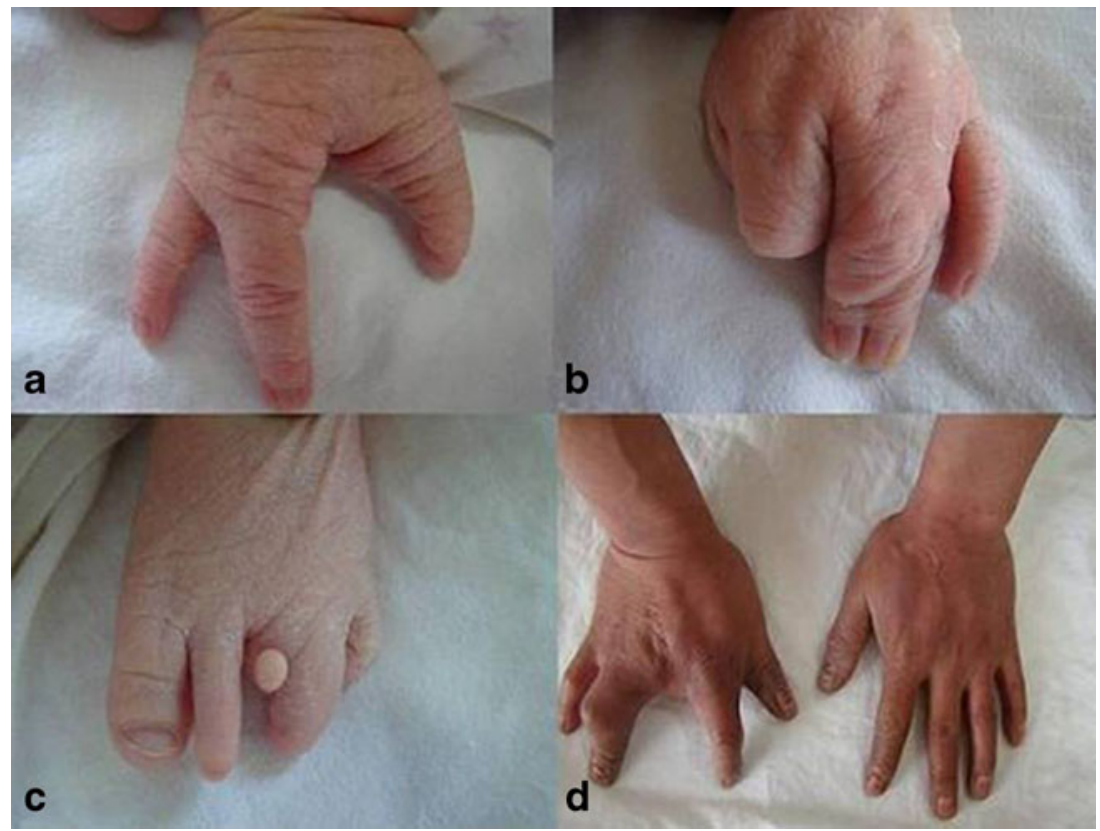


Fig. 4 P63 staining of human cloaca and hindgut in the 4 th week. P63-positive immunoreactions were detected most abundantly on the epithelium of the hindgut, UGS, and CM. (a original magnification $\times 100$; $\mathbf{b}$ original magnification $\times 400)$. $H$ hindgut, URS urorectal septum, $C M$ cloacal membrane, $C L$ cloaca, UGS urogenital sinus

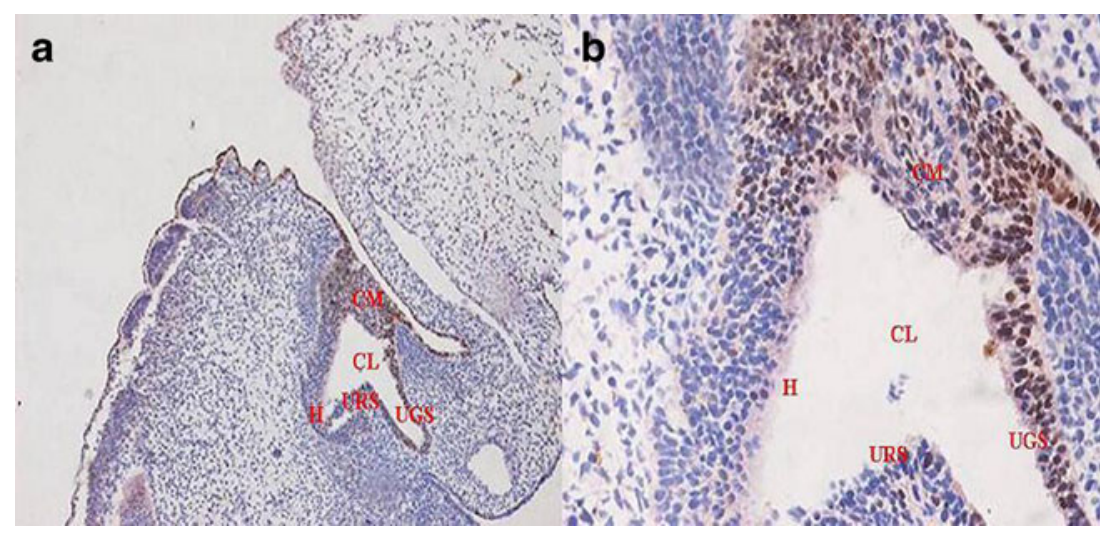

dorsally. Immunoreactivity specific to P63 was detected on the epithelium of the distal-ventral UGS epithelium, URS, hindgut, and cloacal membrane (CM) (Fig. 4).

In the 6th week, the cloacal canal was narrow; however, the fusion of the URS with the CM components was absent. The epithelium of URS, hindgut distal end, and CM were persistently immunoreactive to P63. However, the mucous membrane of the proximal UGS exhibited a weak reaction to P63. Significantly, the CM became thinner and elongated, but the epithelium remained positive until ruptured (Fig. 5).

In the 8th week, the anorectum developed continuously, and the P63 immunoreaction remained strongly immunoreactive on the epithelium of the anal canal and urethra. On the other hand, the mucous membrane of the rectum exhibited no reaction (Fig. 6).

In the 10th week, the expression and distribution of P63 were similar with the pattern during the 9th week. There was considerable proliferation activity within the epithelia of rectum and anal canal. The P63 immunoreaction still remained immunoreactive on the epithelium of the anal canal while the rectum exhibited no reaction (Fig. 7).

\section{Discussion}

The relationship between ARM and SHFM

ARM is a multifactorial disorder for which the individual environmental or genetic risk factors remain largely unknown. A number of syndromes have ARM as one of the possible features and already point to the involvement of specific genes in hindgut development. Among the defined syndromic forms are chromosomal abnormalities (e.g., trisomy 13,18 , and 21 ; cat eye syndrome), monogenic syndromes (e.g., Townes-Rocks, Klippel-Feil, or PallisterHall), and associations (VATER/VACTERL). The recurrence risk for ARM lies within the recurrence risk for the malformation syndrome [1, 2].

In this study, we report on the first Chinese family with intrafamilial clinical variability of SHFM association with ARM. The proband (IV1), now 4 years old, together with his father suffered simultaneously from ARM and SHFM, and none had any of the significant abnormalities of the face, palate, skin, teeth, hair, or nails that characterize the

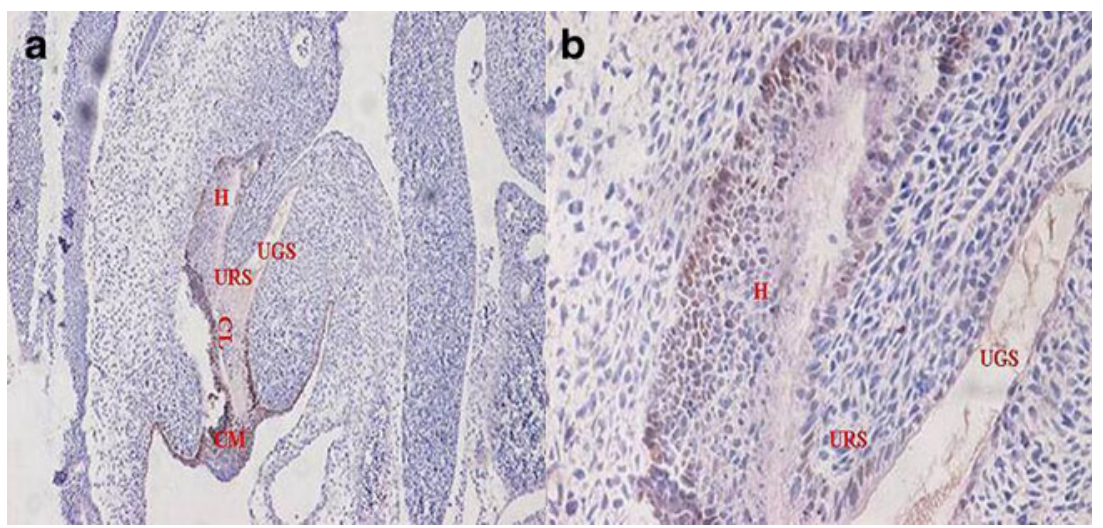

Fig. 5 P63 staining of human cloaca and hindgut in the 6th week. The epithelium of the apical URS and the epithelium of hindgut and CM were strongly and persistently immunoreactive to P63. Significantly, the positive cells of P63 of the ventral epithelium of the URS and the
UGS were decreased (a original magnification $\times 100$; $\mathbf{b}$ original magnification $\times 400$ ). $H$ hindgut, $U R S$ urorectal septum, $C M$ cloacal membrane, $C L$ cloaca, $U G S$ urogenital sinus 
Fig. 6 P63 staining of human anorectum in the 8 th week. The $\mathrm{CM}$ disrupted, the anorectum and the UGS communicated with the amniotic cavity. P63-positive cells disappeared from the epithelium of the rectum. In contrast, a strong positive immunoreaction was displayed in the epithelium of anal canal and urethra (a original magnification $\times 100$; $\mathbf{b}$ original magnification $\times 400$ ). $R$ rectum, $U$ urethra, $A A$ anal canal

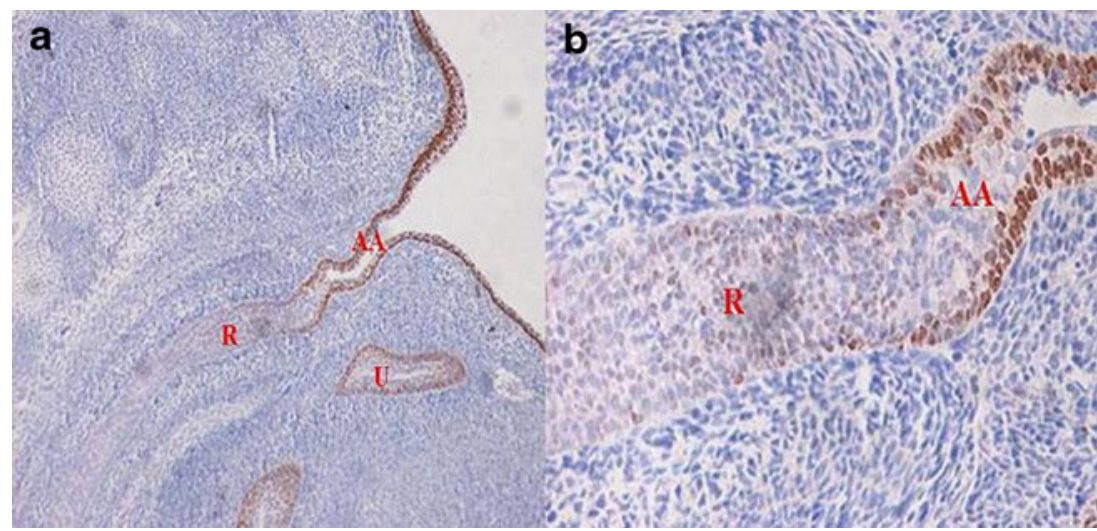

EEC syndrome. To the best of our knowledge, there have been no studies on the simultaneous occurrence of ARM with isolated SHFM.

In previous studies, it was revealed that these candidate genes of SHFM such as HOXD13 [4], DLX/DLX6 [5], and $P 63[6,7]$ could be expressed on the mesenchymal/epithelial cells surrounding the cloacal plate, and downregulation of them resulted in disordered expression of signaling molecules that are involved in the process of hindgut/anorectal development. These molecules include sonic hedgehog $($ Shh $) /$ Gli2 [8, 9] and Bmp7 [10]. Furthermore, in mutant animal analysis, $P 63, D X L 5 / 6$, and $H O X D 13$ knockout mice exhibited severe ARM, such as rectourethral fistula and imperforate anus. These animal studies provided compelling evidence that different genes associated with human SHFM could also be involved in ARM, pointing toward a common molecular origin of these congenital malformations.

Analysis of Arg227Gln P63 mutation in the family

Urogenital defects have been previously reported in P63associated syndromes in conjunction with SHFM. Defects include hypospadias in males, imperforate anus, anteriorly placed and anal atresia, vaginal septum, genital hypoplasia, and micropenis $[11,12]$. In this study, we analyzed the
$D L X 5 / D L X 6, D A C, P 63$, and $H O X D 13$ sequences. A missense mutation was found in the DNA-binding domain of exon 6 in P63: Arg227Gln (797G $\rightarrow A$ ) (Fig. 2). This mutation was not found in the five unaffected individuals in the family. In order to distinguish this mutation from a rare polymorphism, we sequenced 100 unaffected individuals from the general population and were unable to find this substitution. Therefore, we concluded that Arg227Gln of the P63 gene was a vital pathogenic mutation, not merely a polymorphism.

In earlier studies, five frequently mutated amino acids were found in the $P 63$ region in the EEC population: R204, R227, R279, R280, and R304. All were located in CpG islands. These five mutations explained almost $90 \%$ of EEC syndrome patients [13]. Recent genotype-phenotype analysis for the five hotspot mutations revealed significant differences between the corresponding phenotypes. For instance, P63 (R227Q) had been observed in 27 EEC patients and 1 with LMS in which some patients had an anteriorly displaced anus or anal stenosis and hypoplasia of the perineal body. Mutations of this amino acid were rarely associated with orofacial clefting [14-16] and only two had clefts which was striking since approximately $40 \%$ of all are EEC patients. Patients with R227 mutations also had fewer limb defects. Ectrodactyly was present in $40 \%$ (11/28) versus

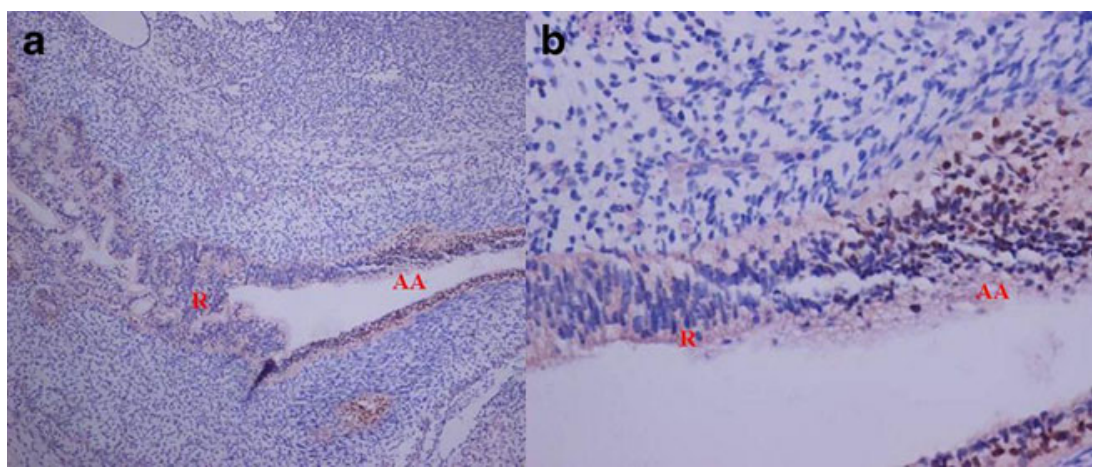

Fig. 7 P63 staining of human anorectum in the 10th week. There was considerable proliferation activity within the epithelia of rectum and anal canal. The P63 immunoreaction still remained strongly immunoreactive on the epithelium of the anal canal while the rectum exhibited no reaction (a original magnification $\times 100$; $\mathbf{b}$ original magnification $\times 400$ ). $R$ rectum, $U$ urethra, $A A$ anal canal 
$68 \%$ for the EEC group as a whole. On the other hand, kidney and urinary problems were quite common. The R227 mutation carriage rate was $11 / 28$ patients ( 40 vs $12 \%$ for EEC patients with other mutations). These characteristics indicated that this mutation differed from other hotspot mutations in terms of function.

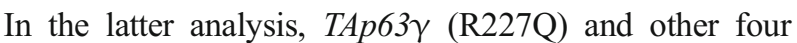
hotspot mutations were localized to the DNA-binding domain and belonged to the EEC syndrome category. However, this mutation was different from the other EEC mutants observed within this syndrome owing to its ability to exert similar effects as the wild type TAp63 $\gamma$ [17]. It was especially interesting to note that the genes involved in development (retinoic acid receptor, alpha (RARA)) was suppressed by this mutant, suggesting the impact of this regulation in relation with the developmental defects associated with the P63 mutant phenotype. The most interesting gene identified was $R A R A$ not only due to its involvement in craniofacial development but also in cloacal development, which was a hallmark feature observed in ARM patients [18].

The role of P63 in human hindgut and anorectal morphogenesis

The aforementioned data had provided impelling evidence that P63 might play an important role in cloacal development and hindgut/anorectal morphogenesis in the animal studies. However, the pattern of expression of $P 63$ had not been investigated with respect to the embryogenesis of the human cloaca, hindgut, or anorectum. Whether P63 was involved in human cloacal development or produces an effect on human embryonic hindgut/anorectal development was unknown. To determine the pattern of expression of $P 63$ and the possible role it played in human hindgut/anorectal morphogenesis, we conducted a systematic study on the spatiotemporal expression of P63 in normal human embryos with special emphasis on embryonic stages from 4th to 9th week gestational age, which was the critical time point for hindgut/anorectal development [19, 20].

The current study showed that the expression of P63 presented a spatiotemporal pattern during human anorectal morphogenesis from 4th to 10th week gestational age. First, P63 expression had time-correlated changes. Specifically, P63positive cells were intensively focused on the epithelium of the distal-ventral UGS epithelium, hindgut, and CM during the dynamic and incessant septation of the cloaca from 4th to 6th week. After the CM ruptured, the positive cells disappeared immediately on the epithelium of the rectum during the 8th week. These data suggested that P63 may accommodate the process of urorectal septation. Furthermore, a spacedependent pattern of P63 was evident in all embryos before the $\mathrm{CM}$ ruptured, which was characterized by dorsoventral patterning. P63 was primarily expressed in the dorsal parts of the cloaca that developed into the anorectum (6th week), whereas in the ventral part of the cloacal epithelium that became the UGS, the expression was weak.

In summary, this study is the first to report on a Chinese family with intrafamilial, clinically variable SHFM in association with ARM without any of the significant abnormalities of the face, palate, skin, teeth, hair, or nails that characterize the EEC syndrome which were found to have an Arg227Gln P63 gene mutation. Furthermore, based on the human embryonic analysis, we determined that the expression of $\mathrm{P} 63$ was persistently active during the dynamic and incessant septation of the cloaca and hindgut, disappearing only after the anus had formed, suggesting that P63 may play a pivotal role in the morphogenesis of the hindgut and anorectum. Further analysis of this cascade reaction's importance on anorectal morphogenesis should facilitate a better understanding of the pathogenesis of ARM.

Acknowledgments This study was supported by the National Natural Science Foundation of China (grant no. 30973141). We would like to thank Hong Gao and Wei Wang (Key Laboratory of Congenital Malformation Research, Ministry of Health, Shengjing Hospital, China Medical University) for their excellent technical assistance.

Dr. Su wrote the first draft of the manuscript and acknowledged support for this work by grants from the National Natural Science Foundation of China (grant no. 30973141).

Open Access This article is distributed under the terms of the Creative Commons Attribution License which permits any use, distribution, and reproduction in any medium, provided the original author(s) and the source are credited.

\section{References}

1. Herman RS, Teitelbaum DH (2012) Anorectal malformations. Clin Perinatol 39(2):403-422

2. Marcelis C, de Blaauw I, Brunner H (2011) Chromosomal anomalies in the etiology of anorectal malformations: a review. Am J Med Genet A 155A(11):2692-2704

3. Blattner A, Huber AR, Röthlisberger B (2010) Homozygous nonsense mutation in WNT1OB and sporadic split-hand/foot malformation (SHFM) with autosomal recessive inheritance. Am J Med Genet A 152A(8):2053-2056

4. Low KJ, Nwbury-Ecob RA (2012) Homozygous nonsense mutation in HOXD13 underlies synpolydactyly with a cleft. Clin Dysmorphol 21(3):141-143

5. Suzuki K, Haraguchi R, Ogata T et al (2008) Abnormal urethra formation in mouse models of split-hand/split-foot malformation type 1 and type 4. Eur J Hum Genet 16(1):36-44

6. Cheng W, Jacobs WB, Zhang JJ et al (2006) $\Delta N p 63$ plays an antiapoptotic role in ventral bladder. Development 133(23):47834792

7. Ching BJ, Wittler L, Proske J et al (2010) P63(TP73L) a key player in embryonic urogential development with significant dysregulation in human bladder exstrophy tissue. Int J Mol Med 26(6):861867

8. Welsh IC, O'Brien TP (2009) Signaling integration in the rugae growth zone directs sequential $S H H$ signaling center formation during the rostral outgrowth of the palate. Dev Biol 336(1):53-67

9. Liu G, Moro A, Zhang JJ et al (2007) The role of Shh transcription activator Gli2 in chick cloacal development. Dev Biol 303(2):448-460 
10. Wu X, Ferrara C, Shapiro E et al (2009) Bmp7 expression and null phenotype in the urogenital system suggest a role in reorganization of the urethral epithelium. Gene Expr Patterns 9(4):224-230

11. Celik TH, Buyukcam A, Simsek-Kiper PO et al (2011) A newborn with overlapping features of AEC and EEC syndromes. Am J Med Genet A 155A(12):3100-3103

12. Ince TA, Cviko AP, Quade BJ et al (2002) P63 coordinates anogenital modeling and epithelial cell differentiation in the developing female urogenital tract. Am J Pathol 161(4):1111-1117

13. Rinne T, Hamel B, van Bokhoven $\mathrm{H}$ et al (2006) Pattern of $P 63$ mutations and their phenotypes - update. Am J Med Genet A 140(13):1396-1406

14. Wei J, Xue Y, Wu L et al (2012) Analysis of large phenotypic variability of EEC and SHFM4 syndromes caused by K193E mutation of the TP63 gene. PLoS One 7(5):e35337

15. Maclean K, Holme SA, Gilmour E et al (2007) EEC syndrome, Arg227Gln TP63 mutation and micturition difficulties: is there a genotype-phenotype correlation? Am J Med Genet Part A 143A(10):1114-1119

16. Sripathomsawat W, Tanpaiboon P, Heering J et al (2011) Phenotypic analysis of Arg227 mutations of TP63 with emphasis on dental phenotype and micturition difficulties in EEC syndrome. Am J Med Genet A 155A(1):228-232

17. Ying $\mathrm{H}$, Chang DL, Zheng $\mathrm{H}$ et al (2005) DNA-binding and transactivation activities are essential for TAp63 protein degradation. Mol Cell Biol 25(14):6154-6164

18. Sasaki Y, Iwai N, Kimura O et al (2011) Establishment of a rescue program for anorectal malformations induced by retinoic acid in mice. J Pediatr Surg 46(7):1396-1399

19. Fritsch H, Aigner F, Ludwikowski B et al (2007) Epithelial and muscular regionalization of the human developing anorectum. Anat Rec 290(11):1449-1458

20. Li FF, Zhang T, Bai YZ et al (2011) Spatiotemporal expression of Wnt5a during the development of the hindgut and anorectum in human embryos. Int J Colorectal Dis 26(8):983-988 